\title{
The activity of comet C/2007 D1 (LINEAR) at 9.7 AU from the Sun ${ }^{\star}$
}

\author{
E. Mazzotta Epifani ${ }^{1}$, M. Dall'Ora ${ }^{1}$, L. Di Fabrizio ${ }^{2}$, J. Licandro ${ }^{3,4}$, P. Palumbo ${ }^{5}$, and L. Colangeli ${ }^{1}$ \\ 1 INAF-Osservatorio Astronomico di Capodimonte, via Moiariello 16, 80131 Napoli, Italy \\ e-mail: epifani@oacn.inaf.it \\ 2 Fundación Galileo Galilei - INAF, Rambla José Ana Fernández Pérez, 7, 38712 Breña Baja, TF, Spain \\ Instituto de Astrofísica de Canarias, c/vía Láctea s/n, 38200, La Laguna, Tenerife, Spain \\ 4 Departamento de Astrofísica, Universidad de La Laguna, 38205 La Laguna, Tenerife, Spain \\ ${ }^{5}$ Universitá Parthenope, Dip. Scienze Applicate, Centro Direzionale Isola C4, 80143 Napoli, Italy
}

Received 23 October 2009 / Accepted 5 January 2010

\section{ABSTRACT}

\begin{abstract}
Aims. We report on the unusual distant activity of the dynamically new comet C/2007 D1 (LINEAR), observed at $r_{\mathrm{h}}=9.7$ AU postperihelion. This comet is particularly interesting because of its quite large perihelion distance $\left(r_{\mathrm{q}}=8.8 \mathrm{AU}\right)$.

Methods. We analyse $V$ and $R$ band images taken at the TNG telescope to characterise the dust coma of the comet and investigate its photometry, colours, and dust production.

Results. The colour of the dust is redder than the $\operatorname{Sun}\left(V-R=0.505 \pm 0.093\right.$ at the photometric aperture of radius $\left.2.2^{\prime \prime}\right)$. The $A f \rho$ value is measured to be $1983 \pm 81 \mathrm{~cm}$ for an aperture of radius $=2.2^{\prime \prime}$. An upper limit to the cometary nucleus radius of $a_{\mathrm{N}} \leq 18 \mathrm{~km}$ is derived. A dust mass-loss rate of $\frac{\mathrm{d} M_{\mathrm{d}}}{\mathrm{d} t}=5.3 \times 10^{2} \mathrm{~kg} \mathrm{~s}^{-1}$ is measured, which implies that $\mathrm{C} / 2007 \mathrm{D} 1$ (LINEAR) is a very active comet despite its quite large perihelion distance.
\end{abstract}

Key words. comets: individual: C/2007 D1 (LINEAR)

\section{Introduction}

Dynamically new comets (DNCs) are those observed on their first passage in the Solar System. They are thought to have formed in the region where giant planets presently orbit (the Jupiter-Neptune zone) and then been scattered towards the Oort cloud region, from which they are occasionally injected towards their first passage close to the Sun. These "new" comets were observed to be intrinsically more active at large distances from the Sun than the periodic ("old") comets (Meech 1988, 1991). Very few DNCs have been studied at heliocentric distances $r$ greater than 5-6 AU, where mechanisms other than the "canonical" sublimation of crystalline water ice must be invoked to explain cometary activity (Meech \& Svoren 2004).

There is debate about the cause of activity at heliocentric distances both too far and cold for significant $\mathrm{H}_{2} \mathrm{O}$ sublimation. Several theoretical models of nucleus thermal evolution and internal differentiation (e.g., Tancredi et al. 1994; Coradini et al. 1997; Prialnik et al. 2004) show that even out to $r=5-6 \mathrm{AU}$ there is sufficient gas flux from sublimating water ice to lift small grains off the surface. Beyond this region, the sublimation of hypervolatiles such as $\mathrm{CO}$ or $\mathrm{CO}_{2}$ has been invoked as the main driver for cometary activity, but it is unlikely that these more volatile ices condense out of the protosolar nebula in a pure form (Meech et al. 2009, and references therein). They will instead be trapped as gas in the cells of amorphous water ice (Laufer et al. 1987; Capria et al. 2000a,b), and post-perihelion activity beyond $5 \mathrm{AU}$ could be caused by perihelion heat wave that

* Based on observations collected at the Italian Telescopio Nazionale Galileo (TNG), operated on the island of La Palma by the Centro Galileo Galilei of the INAF (Istituto Nazionale di Astrofisica) at the Spanish Observatorio del Roque de los Muchachos of the Instituto de Astrofísica de Canarias. penetrates into volatile-rich depths in the nucleus. This causes the amorphous-to-crystalline water ice transition (Notesco et al. 2003; Bar-Nun \& Laufer 2003) and the following release of hypervolatile gas. Anyway, the discovery of active comets that have never been close to the Sun enough to experience significant heating requires a different mechanism.

This is exactly the case for comet C/2007 D1 (LINEAR) (hereinafter C/2007 D1) (Lehmann et al. 2007), which was discovered in February 2007 in the course of the LINEAR (Lincoln Near-Earth Asteroid Research) project (Stokes et al. 2000). It is recognised as a dynamically new comet coming directly from the Oort cloud on an open orbit $(e=1.001)$ with inclination $i=41.5^{\circ}$ and perihelion distance of $q=8.79 \mathrm{AU}$ (passed on June 2007). We observed it in the course of an observational program designed to investigate the distant dust environment of a number of long period comets at very large heliocentric distances, beyond the "water" zone.

\section{Observations and data reduction}

The comet C/2007 D1 was observed on May 29, 2009 with the $3.52 \mathrm{~m}$ Telescopio Nazionale Galileo (TNG) at the Observatorio del Roque de los Muchachos (La Palma, Canary Islands), when it was at the heliocentric distance of $r=9.7 \mathrm{AU}$ and the geocentric distance of $\Delta=9.33 \mathrm{AU}$. The comet was at the phase angle $\alpha=5.69^{\circ}$ and had a position angle (PA) of the extended Sun-target radius vector of $117.5^{\circ}$.

The images were obtained with the DOLORES (Device Optimised for the LOw RESolution) instrument, a focal reducer instrument installed at the Nasmyth B focus of the TNG. The detector is a $2048 \times 2048$ E2V 4240 thinned back-illuminated, deep-depleted, Astro-BB coated CCD with a pixel size of 
Table 1. Photometry, Af $\rho$, and colours of the comet C/2007 D1 (LINEAR).

\begin{tabular}{cccccccc}
\hline \hline$\phi\left[^{\prime \prime}\right]^{a}$ & $\log _{10} \rho[\mathrm{km}]^{b}$ & $V$ & $R$ & $V-R$ & $A f \rho[\mathrm{cm}]^{c}$ & $A f \rho[\mathrm{cm}]^{d}$ & Reddening $[\% / 1000 \AA]$ \\
\hline 0.5 & 3.531 & $21.723 \pm 0.123$ & $21.008 \pm 0.092$ & $\ldots$ & $\ldots$ & $\ldots$ & $\ldots$ \\
1.1 & 3.832 & $20.468 \pm 0.072$ & $19.861 \pm 0.060$ & $0.607 \pm 0.132$ & $1400 \pm 93$ & $1774 \pm 97$ & $1.91 \pm 1.12$ \\
2.2 & 4.133 & $19.493 \pm 0.049$ & $18.988 \pm 0.044$ & $0.505 \pm 0.093$ & $1719 \pm 78$ & $1983 \pm 81$ & $1.19 \pm 0.80$ \\
3.3 & 4.309 & $19.057 \pm 0.042$ & $18.584 \pm 0.039$ & $0.473 \pm 0.080$ & $1712 \pm 65$ & $1917 \pm 68$ & $0.98 \pm 0.67$ \\
\hline Sun & & \multicolumn{5}{c}{$0.35^{e}$} &
\end{tabular}

Notes. ${ }^{(a)}$ Radius of the photometry aperture. ${ }^{(b)}$ Linear radius of the aperture at the comet. ${ }^{(c)}$ In the $V$ filter. ${ }^{(d)}$ In the $R$ filter. ${ }^{(e)}$ Colour of the Sun is in the modern Johnson-Cousin system used by Landolt (1992) (from Holmberg, Flynn \& Portinari 2006).

$13.5 \mu \mathrm{m}$. The scale is $0.252 \mathrm{arcsec} / \mathrm{px}$, which yields a field of view of about $8.6 \times 8.6$ arcmin. The instrument was equipped with the broadband filters $V$ and $R$ of the Johnson-Cousin system.

Several long (600 s) exposures were obtained for the object, the telescope tracking at the non-sidereal rate corresponding to the predicted motion of the comet, with the following sequence: $R R R R R V V V V V R R R V V V$, for a total of $8 R$ and $8 V$ exposures. All the images were corrected for overscan, bias, and flat-fielding in the standard manner, using tools available in $\mathrm{IRAF}^{1}$. The overscan level was estimated from the overscan section of each scientific image, while the bias value was obtained from an average of several zero-exposure frames, each of them being corrected for the overscan, taken at the beginning of the night. A masterflat for each filter was obtained by averaging of several twilight exposures, individually corrected for overscan and bias. To optimize the observing time spent on the scientific targets, standard fields were observed at only a single airmass to derive the photometric zero-points, while extinction coefficents were estimated directly from the scientific frames, which cover an airmass range between 1.13 and 1.50 , and between 1.06 and 1.37 in the $V$ and $R$ bands, respectively. To this aim, aperture stellar photometry was performed on each scientific image using the DAOPHOT/ALLSTAR package (Stetson 1987). Instrumental magnitudes were therefore compared with DAOMATCH/DAOMASTER (Stetson 1993). This package computes a robust weighted average zero-point difference between individual frames: since all the images in a given filter have the same exposure time, the differences in the zeropoint are mainly related to the different airmass. The slope of the instrumental zero points plotted against the airmass therefore corresponds to the extinction coefficient. Photometric zeropoints were finally computed from the Landolt's fields SA104 350 and PG1047 (Landolt 1992).

Despite the very large heliocentric distance, C/2007 D1 was easily identificable and appeared active in all the single exposures. To increase the SNR for the image analysis, we decided to coadd the $R$ and $V$ images in order to obtain two "final" images for each filter, one for the sequence of the first 5 and one for the last 3 exposures. We decided not to combine all the 8 images into one since the comet significantly changed its airmass during the whole sequence (see above). The position of C/2007 D1 optocentre in each single image selected for the coadding was determined by fitting a two-dimensional Gaussian to the innermost (64 and 81 for the $R$ and $V$ filter, respectively) pixels of the coma. The images were then recentred using this optocentre and summed. Sky correction was performed by subtracting a first-order polynomial sky approximation computed from the

\footnotetext{
${ }^{1}$ IRAF is distributed by the National Optical Astronomical Observatory, which is operated by the Association of Universities for Research in Astronomy, Inc., under cooperative agreement with the National Science Foundation.
}

pixel areas containing no stars. The image processing and analysis was performed with the aid of the ESO's Munich Image Data Analysis System (MIDAS 1998). Two examples of the final coadded images are shown in Fig. 1 (obtained from the first 5 exposures for both filters).

\section{Observational results}

Despite its large heliocentric distance at the time of observation $(r=9.7 \mathrm{AU})$, the comet $\mathrm{C} / 2007 \mathrm{D} 1$ appears to be a very active object with a well-developed coma and a broad tail-like structure, extending in the S-E quadrant approximately in the anti-solar direction (PA of the comet: $117.5^{\circ}$ ). The final images obtained in both filters were processed using a Laplacian filtering (Boehnhardt \& Birkle 1994) to search for any non-spherical structure (e.g., jets, fans) in the coma morphology. This method is sensitive to gradient changes on different scales depending on the width of the spatial filter applied. No clear structure was found, as shown in Fig. 1 for two of the obtained final (coadded) images.

The magnitudes of the comet, derived in different optical apertures centred in the optocentre, are summarised in Table 1. For each photometric aperture, these magnitude values are obtained as a weighted mean between the two values derived from the 2 images (coadditions of the first 5 exposures and the last 3 ). The photometric $R$ magnitude can be used to estimate the upper limit to the geometric cross-section of the cometary nucleus, using the expression of Russell (1916), derived for asteroids observed at large phase angle and conveniently reformulated by Jewitt (1991), which, in the case of a spherical object, is given by

$A a_{\mathrm{N}}^{2} \leq 2.24 \times 10^{22} r^{2} \Delta^{2} 10^{0.4\left(m_{\odot}-R+\beta \alpha\right)}$

where $A$ is the geometric albedo, $a_{\mathrm{N}}[\mathrm{m}]$ is the radius of the target, $m_{\odot}$ is the magnitude of the Sun in the same wavelength band of observations $\left(m_{\odot}=-27.10\right.$, see Holmberg et al. 2006), and $\alpha$ and $\beta$ are the phase angle $\left[{ }^{\circ}\right]$ and the phase coefficient $\left[\mathrm{mag} /{ }^{\circ}\right]$, respectively, in the phase darkening function $\Phi(\alpha)=10^{-0.4 \alpha \beta}$. We assumed that the nucleus is a point-like source embedded in an extended coma, therefore we can sample the nucleus contribution (plus a small contribution from the near-nucleus coma) using all the flux inside the photometric aperture corresponding to the stellar point-spread-function (PSF) of the image. We computed the FWHM of the PSF, i.e., the average for several stars in all the (standard) frames, to be of radius $\phi=0.5^{\prime \prime}$ : within this photometric aperture, the $R$ magnitude of the comet is $R=21.008 \pm 0.092$ (see Table 1). In Eq. (1), we decided to adopt the classical value of geometric albedo $A=0.04$ (commonly assumed for short period comets), also to be consistent with a similar study by Meech et al. (2009) on very distant comets. This value is similar to the (few) measured albedo values for other long period comets such as C/1983 H1 (0.03 \pm 0.01 , Lamy et al. 2004), C/2001 OG108 (0.03 \pm 0.005 , Abell et al. 2003), and C/1985 O1 (0.04 \pm 0.03 , Jorda et al. 2000). For 

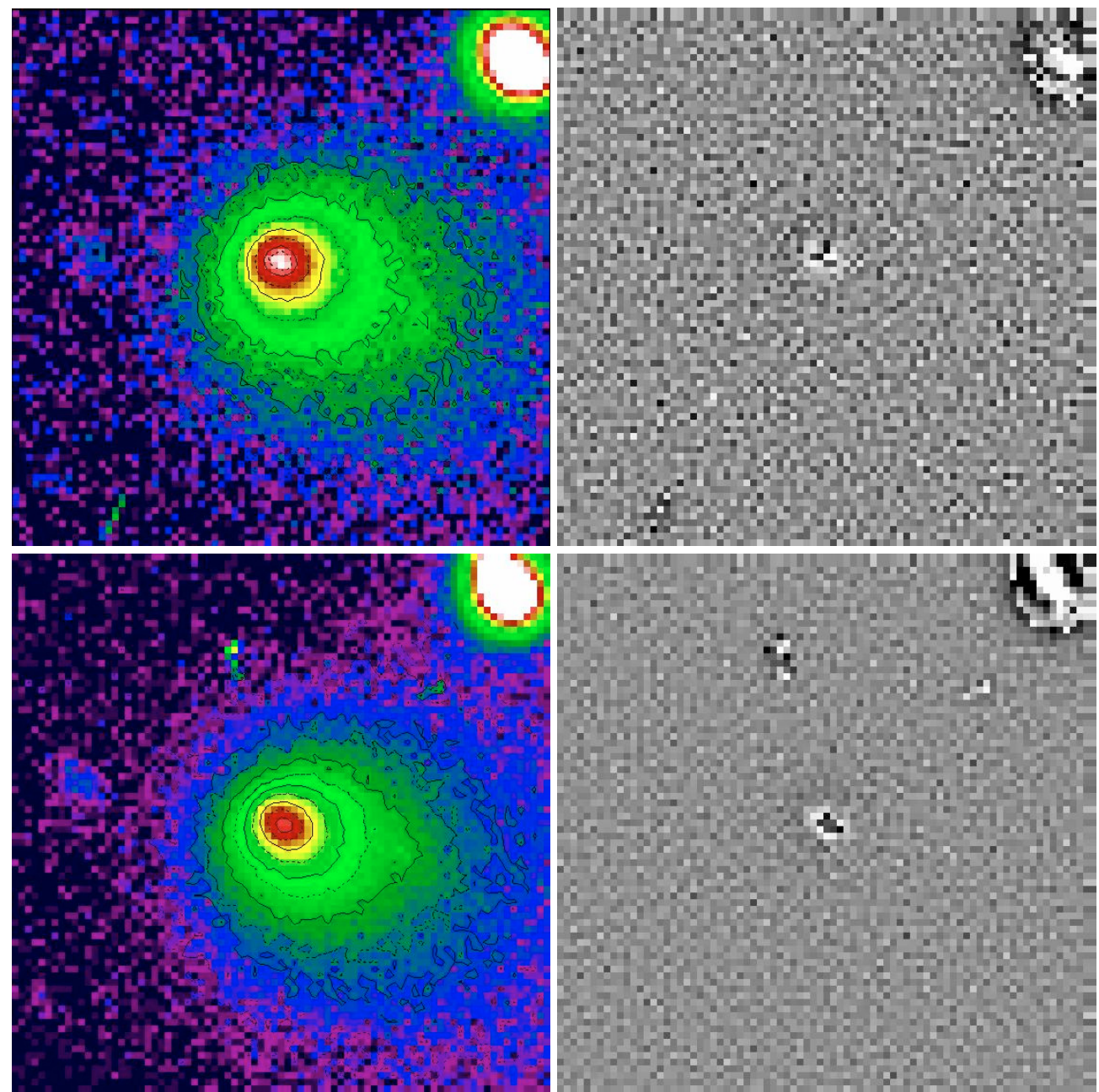

Fig. 1. Coadded images of the comet C/2007 D1 (LINEAR) (sequence of first 5 single images, see text) in the $V$ (top) and $R$ (bottom) filters, together with the corresponding processed images with Laplace filter (right panels) to enhance any high frequency spatial structure. The linear scale for all images is $6.8 \times 10^{4} \mathrm{~km}$. N is up, E is right. The grey scale is logarithmic. The faintest plotted isophote corresponds to 25.37 and 25.12 mag $\operatorname{arcsec}^{-2}$ for the $V$ and $R$ filters, respectively.

the phase coefficient $\beta$, we decided to use in Eq. (1) the value $\beta=0.045 \mathrm{mag} /{ }^{\circ}$, which is the average of the (few) measured phase coefficient values (values for 8 short period comets in the range $0.025-0.06 \mathrm{mag} /{ }^{\circ}$, see Lamy et al. 2004). With the above values, Eq. (1) results in a $a_{\mathrm{N}} \leq 18 \mathrm{~km}$ for comet C/2007 D1.

The data obtained during the observations allow us to perform an analysis (at least partially) of the coma colours of $\mathrm{C} / 2007$ D1. Table 1 summarises the $(V-R)$ colours obtained at different optical apertures centred in the optocentre. The dust colour of the coma is slightly redder than the Sun at all nucleus distances, and is $0.505 \pm 0.093$ at the photometric aperture of $\phi=2.2^{\prime \prime}$. The colour of the dust can also be computed in terms of the reddening (see e.g. Lara et al. 2003), which indicates the percentage of change in the strength of the continuum per $1000 \AA$ (when far from the Sun, the broadband filters $V$ and $R$ used here are relatively free from strong gas emission bands) given by

reddening $=\frac{1}{A f \rho} \frac{(A f \rho)^{\prime}-(A f \rho)}{\lambda^{\prime}-\lambda}$

where $A f \rho$ (see below) is computed at wavelenghts $\lambda=527 \mathrm{~nm}$ and $\lambda^{\prime}=644 \mathrm{~nm}$ (band center of the TNG $V$ and $R$ filters, respectively). Values of reddening obtained at different optical apertures centred on the optocentre are listed in Table 1: they confirm the picture of a neutral to slightly reddish dust coma.

The $A f \rho$ value [cm] (A'Hearn et al. 1984), where $A$ is the average grain albedo, $f$ the filling factor in the aperture field of view, and $\rho$ the linear radius of the aperture at the comet, i.e., the sky-plane radius, is usually used as a proxy for the cometary dust production. When the cometary coma is in a steady-state, $A f \rho$ is an aperture-independent parameter. It is formulated to take into account the solar flux scattered by the cometary dust towards the observer, and can be derived from the calculated photometric dust coma magnitude

$A f \rho=\frac{4 \cdot r^{2} \cdot \Delta^{2} \cdot 10^{0.4\left(m_{\odot}-R\right)}}{\rho}$

where $m_{\odot}$ is the Solar magnitude in the $R$ band. Values of $A f \rho$ for comet C/2007 D1, in different aperture radii, are shown in Table 1. A monotonical decrease in $A f \rho$ values with the nucleocentric distance $\rho$ may indicate non-steady-state dust emission and possibly dust grain fading or destruction, as reported for several comets, such as C/2000 WM1 (LINEAR) (Lara et al. 2004) and C/2001 Q4 (NEAT) (Tozzi et al. 2003). In contrast, for an ideal steady-state coma, $A f \rho$ should be constant at all apertures. Values for comet C/2007 D1, reported in Table 1, slightly differ at different cometocentric distances but are quite compatible 
Table 2. Model dust mass loss-rate of comet C/2007 D1 (LINEAR).

\begin{tabular}{cccccc}
\hline \hline$m_{\mathrm{d}}{ }^{a}$ & $C_{\mathrm{d}}\left[\mathrm{m}^{2}\right]^{b}$ & $M_{\mathrm{d}}[\mathrm{kg}]^{c}$ & $v(r)\left[\mathrm{m} \mathrm{s}^{-1}\right]^{d}$ & $\tau[\mathrm{s}]^{e}$ & $\frac{\mathrm{d} M_{\mathrm{d}}}{\mathrm{d} t}\left[\mathrm{~kg} \mathrm{~s}^{-1}\right]^{f}$ \\
\hline 18.71 & $4.69 \times 10^{9}$ & $1.98 \times 10^{8}$ & $50^{\dagger}$ & $3.74 \times 10^{5}$ & $5.30 \times 10^{2}$ \\
& & & $466^{\S}$ & $4.00 \times 10^{4}$ & $4.94 \times 10^{3}$ \\
\hline
\end{tabular}

Notes. ${ }^{(a)}$ Coma magnitude in the projected annulus between $\phi=0.5^{\prime \prime}$ and $\phi^{\prime}=3.3^{\prime \prime} .{ }^{(b)}$ Derived dust cross-section. ${ }^{(c)}$ Derived dust mass in the coma annulus. ${ }^{(d)}$ Dust grains radial outflow speed in the coma annulus. $\dagger$ Realistic case. $\S$ Upper limit case (gas outflow velocity). ${ }^{(e)}$ Crossing time of the (projected) coma annulus. ${ }^{(f)}$ Derived dust mass loss rate.

within errors, and a clear monotonical trend is not easily identified. Therefore, we can conclude that the dust environment of the comet is consistent with a scenario of steady-state emission.

The very high values of the cometary Af $\rho$ (e.g., $1983 \pm$ $81 \mathrm{~cm}$ for an aperture of $\phi=2.2^{\prime \prime}$ ) are consistent with a very active object. To obtain a first-order quantitative estimate of the dust mass-loss rate by comet C/2007 D1, we decided to apply the same method used by Jewitt (2009) to compute the dust production rate of active Centaurs in the region between 5 and 12 AU.

The method consists of computing the apparent magnitude of the pure coma $m_{\mathrm{d}}$ in an annulus between the inner $\phi$ (as defined above) and a conveniently chosen outer $\phi^{\prime}$ as $m_{\mathrm{d}}=$ $-2.5 \log \left(10^{-0.4 R}-10^{-0.4 R^{\prime}}\right)$, where $R$ and $R^{\prime}$ are the magnitudes within apertures of radii $\phi$ and $\phi^{\prime}$, respectively, and then computing the total cross-section of the coma dust particles in the projected annulus. A value of $\phi^{\prime}=3.3^{\prime \prime}$ was adopted, derived from the aperture photometry applied to the final (coadded) images. The dust cross-section $C_{\mathrm{d}}$ derived from the photometry is related to the dust mass $M_{\mathrm{d}}$ by means of the dust size distribution, for which a good approximation is obtained by setting a power law distribution with $q=3.5$ in a dust grain size range between $a_{-}=0.1 \mu \mathrm{m}$ and $a_{+}=1 \mathrm{~cm}$ (Grün et al. 2001). The relation is given by $M_{\mathrm{d}}=\frac{4}{3} \rho\left(a_{-} a_{+}\right)^{1 / 2} C_{\mathrm{d}}$, where the size of the average dust grain scatterer is $\langle a\rangle=\left(a_{-} a_{+}\right)^{1 / 2} \sim 30 \mu \mathrm{m}$, consistent with the observation that the dust coma of C/2007 D1 is reddish compared to the Sun (see above).

Following Jewitt (2009), the dust-loss rate is derived by considering the time of residence $\tau(r)$ of dust grains in the (projected) annulus between $\phi$ and $\phi^{\prime}$ equal to

$\tau(r)=1.5 \times 10^{11} \Delta\left(\phi^{\prime}-\phi\right) / v(r)$

where $\phi$ and $\phi^{\prime}$ are expressed in radians, and $v(r)$ is the radial outflow speed of dust grains from the nucleus. Expansion measurements for comet Hale-Bopp in the range $4 \leq r \leq 14 \mathrm{AU}$ infer that $v(r)=v_{0} \cdot\left(r_{0} / r\right)^{1 / 4}$, where $v_{0}=550 \mathrm{~m} \mathrm{~s}^{-1}$ and $r_{0}=5 \mathrm{AU}$ (Biver et al. 2002). This expression clearly provides a simplified estimation of (and an upper limit to) the dust-grain flow speed $v(r)=466 \mathrm{~m} \mathrm{~s}^{-1}$, since the Hale-Bopp measurements refer to gas. Dust grains probably travel more slowly than gas, since they may not be perfectly coupled with it (the coupling efficiency also depending on their size), and do not travel radially across the projected coma annulus. Probstein's theory (Probstein 1969; Fulle et al. 1998) predicts, for spherical dust grains emitted from a perfectly homogeneous nucleus, a dust velocity close to $10 \%$ of the gas value. Adopting then a more realistic dust grain velocity $v(r) \sim 50 \mathrm{~m} \mathrm{~s}^{-1}$, a value of $\frac{d M_{\mathrm{d}}}{\mathrm{d} t}=5.3 \times 10^{2} \mathrm{~kg} \mathrm{~s}^{-1}$ is obtained from the photometric model. Table 2 summarises the values derived for all the above defined quantities, for both the realistic value and the upper limit (gas ouflow) of the dust grain velocity.

\section{Discussion and conclusions}

The results described in the previous section are consistent with the properties of a very active comet. When considering a proxy for the dust production rate, the comet C/2007 D1 is found to have an $A f \rho$ value of the same order or even larger than that measured at lower heliocentric distances for some of the more active LPCs: e.g., C/2003 O1 (LONEOS), from $\sim 600$ to $\sim 1300 \mathrm{~cm}$ at 7.02 AU (Mazzotta Epifani et al. 2009; Meech et al. 2009), and C/2001 G1 (LONEOS), $1800 \mathrm{~cm}$ at $8.3 \mathrm{AU}$ (Meech et al. 2009).

The first-order photometric model (Jewitt 2009) for the determination of the dust-loss rate that we applied to the images, by using a realistic value of the dust grain velocity (see above), inferred a quite high value of $\frac{\mathrm{d} M_{\mathrm{d}}}{\mathrm{d} t}=5.3 \times 10^{2} \mathrm{~kg} \mathrm{~s}^{-1}$. This value is indicative of a very active comet, similar to the "paradigm" comet C/1995 O1 (Hale-Bopp), for which a $\frac{\mathrm{d} M_{\mathrm{d}}}{\mathrm{d} t} \sim 500 \mathrm{~kg} \mathrm{~s}^{-1}$ was obtained by modelling at $r=13$ AU (Fulle et al. 1998). For comparison, by applying the first-order photometric model to the active Centaur 29P/S-W 3 at $r=5.8$ AU, Jewitt (2009) obtained a dust production rate of $\frac{\mathrm{d} M_{\mathrm{d}}}{\mathrm{d} t}=5.1 \times 10^{3} \mathrm{~kg} \mathrm{~s}^{-1}$, while the application of the inverse tail model by Fulle et al. (1992), which reconstructed the dynamical dust environment (ejection velocity, dust-loss rate, and reliable dust-grain size-distribution) of the Centaur, resulted in a constant value of the dust production rate of $6 \times 10^{2} \mathrm{~kg} \mathrm{~s}^{-1}$ during the three years spent by the Centaur at about the same heliocentric distance. To a first-order approximation, if we apply the same scaling factor of an order of magnitude to the upper limit to the dust-loss rate $\left(\frac{\mathrm{d} M_{\mathrm{d}}}{\mathrm{d} t}=4.94 \times 10^{3}\right.$, see Table 2), we would obtain for comet C/2007 D1 a dust-loss rate of $\sim 500 \mathrm{~kg} \mathrm{~s}^{-1}$, very similar to the value obtained using the photometric model.

We conclude that the post-perihelion activity of comet C/2007 D1 is very intense, despite, in its recent orbital history, it never having passed at heliocentric distances smaller than $r=8.8 \mathrm{AU}$. This poses interesting constraints on theoretical models that should be able to explain the distant activity of minor bodies orbiting in the outer part of the Solar System.

Acknowledgements. We gratefully acknowledge funding from Italian Space Agency (ASI) under contract I/015/07/0. J.L. gratefully acknowledges support from the Spanish "Ministerio de Ciencia e Innovación” project AYA2008-06202C03-02.

\section{References}

Abell, P. A., Fernandez, Y. R., Pravec, P., et al. 2003, in Lunar and Planetary Science XXXIV, Abstract \# 1253 (Houston: Lunar and Planetary Institute) A'Hearn, M. F., Schleicher, D. G., Feldman, P. D., Millis, R. L., \& Thompson, D. T. 1984, AJ, 89,579

Bar-Nun, A., \& Laufer D. 2003, Icarus, 161, 157

Biver, N., Bockelée-Morvan, D., Colom, P., et al. 2002, EM\&P 90, 1, 5

Boehnhardt, H., \& Birkle, K. 1994, A\&A, 107, 101

Capria, M. T., Coradini, A., De Sanctis, M. C., \& Orosei, R. 2000a, A\&A, 357, 359 
Capria, M. T., Coradini, A., De Sanctis, M. C., \& Orosei, R. 2000b, AJ, 119, 3112

Coradini, A., Capaccioni, F., Capria, M. T., et al. 1997, Icarus, 129, 337

Fulle, M. 1992, Nature, 359, 6390, 42

Fulle, M., Cremonese, G., \& Bóhm, C. 1998, AJ, 116, 1470

Grün, E., Hanner, M. S., Peschke, S. B., et al., 2001, A\&A, 377, 1098

Holmberg, J., Flynn, C., \& Portinari, L. 2006, MNRAS, 367, 449

Jewitt, D. C. 1991, in Comets in the Post-Halley Era, ed. R. L. Newburn, M.

Neugebauer, \& J. Rahe (Dordrecht: Kluwer Acad. Publ.), 1

Jewitt, D. C. 2009, AJ, 137, 4296

Jorda, L., Lamy, P., Groussin, O., et al. 2000, in Proceedings of ISO Beyond

Point Sources: Studies of Extended Infrared Emission, ed. R. J. Laureijs et al., ESA-SP, 455, Noordwijk, The Netherlands

Lamy, P. L., Toth, I., Fernández, Y. R., \& Weaver, H. A. 2004, in Comets II, ed. M. Festou, H. U. Keller, \& H. A. Weaver (Tucson: Univ. Arizona Press)

Landolt, A. U. 1992, AJ, 104, 1, 340

Lara, L.-M., Licandro, J., Oscoz, A., \& Motta, V. 2003, A\&A, 399, 763

Lara, L.-M., Tozzi, G. P., Boehnhardt, H., DiMartino, M., \& Schulz, R. 2004, A\&A, 422, 717

Laufer, D., Kochavi, E., \& Bar-Nun, A. 1987, Phys. Rev. B, 36, 9219

Lehmann, G., Ticha, J., Tichy, M., et al. 2007, MPEC, 2007-D22
Mazzotta Epifani, E., Palumbo, P., Capria, M. T., et al. 2009, A\&A, 502, 355 Meech, K. J. 1988, BAAS, 20, 835

Meech, K. J. 1991, in Comets in the Post-Halley Era, ed. R. L. Newburn Jr., M. Neugebauer, \& J. Rahe (Kluwer Acad. Pub.)

Meech, K. J., \& Svoren, J. 2004, in Comets II, ed. M. Festou, H. U. Keller, \& H. A. Weaver (Tucson: Univ. Arizona Press)

Meech, K. J., Pittichova, J., Bar-Nun, A., et al. 2009, 201, 2, 719

Notesco, G. A., Bar-Nun, A., \& Owen, T. 2003, Icarus, 162, 183

Prialnik, D., Benkhoff, J., \& Podolak, M. 2004, in Comets II, ed. M. Festou, H. U. Keller, \& H. A. Weaver (Tucson: Arizona Press)

Probstein, R. F. 1969, in Problems of Hydrodynamics and Continuum Mechanics, ed. F. Bisshopp et al. (Philadelphia: Soc. Indust. Appl. Math.), 568

Russell, H. N. 1916, ApJ, 43, 173

Stetson, P. B. 1987, PASP, 99, 191

Stetson, P. B. 1993, Stellar Photometry - Current Techniques and Future Developments, IAU Colloq., 136, 291

Stokes, G. H., Evans, J. B., Viggh, H. E. M., Shelly F. C., \& Pearce E. C. 2000, Icarus, 148, 21

Tancredi, G., Rickman, H., \& Greemberg, J. M. 1994, A\&A, 286, 659

Tozzi, G. P., Boehnhardt, H., \& Lo Curto, G. 2003, A\&A, 398, L41 